\title{
Pharmakovigilanz in der Phytotherapie: Vorsorge ja, Missbrauch nein
}

Pharmakovigilanz bedeutet laut WHO das Analysieren und Abwehren von Arzneimittelrisiken. Dafür gibt es gute Gründe: Etwa 3-6\% aller Krankenhaus-Einweisungen und ca. 5-9\% der Klinikkosten sollen auf unerwünschte Arzneimittelwirkungen (UAWs) zurückgehen (1). Vieles steht dabei noch im Konjunktiv, denn die genaue Erfassung ist schwierig. Seltene UAWs werden mit randomisierten klinischen Studien (RCTs) kaum entdeckt. Auch die Erhöhung der Fallzahlen um etwa den Faktor 10, wie sie mit Anwendungsbeobachtungen (AWBs) erreicht wird, bringt bei weitem noch nicht alles ans Licht. Das wichtigste Frühwarnsystem für seltenere Risiken beruht daher auf den Spontanmeldungen von Ärzten und Apothekern.

Die Wächter der Pharmakovigilanz beklagen jedoch zu niedrige Meldequoten, die selbst bei schweren UAWs nur bei 5-10\% liegen sollen (1). Den Ärzten und Apothekern vor Ort missfällt auf der anderen Seite die Form der Rückmeldung, die sich gewöhnlich in Endloslisten von Einzelmeldungen oder Riesendiagrammen über 30 oder mehr Organsysteme erschöpft. Das ist nützlich für die Vigilanzbuchhaltung, aber wertlos für die Praktiker. Deren Therapieentscheidung wird durch deskriptive Aufbereitungen dieser Art nicht erleichtert. Mehr Hilfen zum Handeln böten relative Häufigkeiten von UAWs, die im direkten Vergleich zu den jeweiligen therapeutischen Alternativen stünden.

Gezielte klinische oder pharmako-epidemiologische Studien, wie sie beispielhaft in diesem Heft auf den Seiten 178 bis 182 referiert werden, können in Einzelfällen Hinweise zur relativen Häufigkeit geben; zum systematischeren Vergleich des Gesamtrisikos sind sie aber auch nicht geeignet. Zu diesem Zweck werden gegenwärtig in Deutschland Pharmakovigilanz-Zentren etabliert. Deren Fokus richtet sich aber vorwiegend auf UAW-bedingte Klinikeinweisungen, die wiederum mit Phytopharmaka so gut wie nie vorkommen. Damit stützt sich die Phyto-Pharmakovigilanz im Wesentlichen auf das spontane Meldesystem, zu dessen quantitativer Bewertung es jedoch in einer Broschüre der Arzneimittelkommission der deutschen Ärzteschaft (AKdÄ) heißt: »Da es keine genauen Daten zur Anzahl der Personen gibt, die einen Wirkstoff einnehmen, ist es nicht möglich, Angaben zur absoluten Häufigkeit bestimmter Risiken, zur Inzidenz einer UAW oder zum relativen Risiko zu machen« (1).
Verbrauchsbezogenen Berechnungen des relativen Risikos, wie sie der Praktiker braucht, wird damit eine Absage erteilt. Anstelle des quantitativen Gruppenvergleichs tritt die qualitative Bewertung des Einzelfalles. Dafür gibt es aber weder allgemeingültige Regeln noch validierte Methoden. Die Auslegung der Berichte hängt in hohem Maß vom Ermessen des einzelnen Gutachters ab. Darin liegt zugleich ein Freibrief für Interessensvertreter, die pflanzliche Arzneimittel lieber heute als morgen vom Markt sähen. Anlass genug, zu prüfen, ob alles zutrifft, was die Phytotherapie in derart prekäre Abhängigkeiten bringt. Stimmt es denn tatsächlich, dass es »keine genauen Daten zur Anzahl der Personen gibt, die einen Wirkstoff einnehmen«? Werden nicht alljährlich die zu Lasten der gesetzlichen Krankenversicherung (GKV) verbrauchten definierten Tagesdosen (DDD) aller Arzneimittel im »Arzneiverordnungs-Report» (2) publiziert. Gibt es nicht zusätzlich auch Verbrauchszahlen des Institutes für Medizinische Statistik, Frankfurt (IMS), bei denen auch die frei verkäuflichen Arzneimittel mit erfasst sind? Was resultiert, wenn man auf dem Boden solcher Zahlen quantitative Risikovergleiche zu konkurrierenden Wirkstoffgruppen durchführt? Dem Autor dieses Editorials fiel durch Zufall die Probe aufs Exempel zu und die verlief so:

Der Chefredakteur der AKdÄ-Zeitschrift „Arzneiverordnung in der Praxis» bat im Oktober 2005 um einen Beitrag über Wechselwirkungen mit Ginkgobiloba-Extrakt. Der Artikel sollte präzise aber praxisnah sein. Aussagen zu den Risiken sollten in möglichst knappen Zahlen, zum Beispiel in Form der number needed to harm (NNH) zusammengefasst werden. Die Gesamtbewertung sollte den Kostenaspekt nicht ausklammern. Im redaktionellen Fortgang wurde hinsichtlich der Praxisnähe Einigkeit darüber erzielt, einen direkten Vergleich zu der von der AKdÄ empfohlenen therapeutischen Alternative mit einzubeziehen, nämlich zu den Acetylcholinesterasehemmern $(\mathrm{ACH})$. Anstelle der nicht berechenbaren $\mathrm{NNH}$ durfte eine number needed to report (NNR) treten, worunter diejenigen Behandlungszahlen in beiden Stoffgruppen zu verstehen sind, die jeweils einen spontanen Meldefall auslösten.

Das Ergebnis war überraschend: Laut "Arzneiverordnungs-Report» wurden im Zeitraum von 19902004 insgesamt 2475 Mio. DDD von Ginkgo-bilobaExtrakt zulasten der GKV verordnet. Die AkdÄ erhielt im gleichen Zeitraum insgesamt 239 UAW- 
Meldefälle. Daraus resultieren 2475 Mio. : $239=$ 10356000 DDD pro Meldefall. Das bedeutet bei 1-jähriger Therapie 10356000 : 365 = 28373 Behandlungszyklen pro Meldefall. Für die Gruppe der ACH wurden laut »Arzneiverordnungs-Report» von 1999-2004 insgesamt 73 Mio. DDD zulasten der GKV verordnet. Die AkdÄ erhielt im gleichen Zeitraum insgesamt 598 Meldefälle. Daraus resultieren: 73 Mio. : $598=122000$ DDD pro Meldefall oder bei 1-jähriger Therapie 122000 : 365 = 334 Patientenbehandlungszyklen pro Meldefall. Gegenüber den Ginkgo-biloba-Extrakten ergibt sich daraus ein 28373 : 334 = 85-fach höheres Risiko des Auftretens meldepflichtiger UAWs, das Ganze bei 5fach höheren Behandlungskosten unter den $\mathrm{ACH}$.

Vergleiche dieser Art sind von zusätzlichen Faktoren abhängig, z.B. vom Zeitpunkt der Einführung des Arzneimittels, von der Compliance der Patienten oder auch von Medienberichten. Solche Einflüsse müssen angemessen geprüft und diskutiert werden. So auch geschehen in dem Artikel für die AKdÄZeitschrift. Deren Chefredakteur war trotz des anders erwarteten Ergebnisses von der Arbeit sehr angetan und wollte den Beitrag unverändert publizieren. Andere Mitglieder der AKdÄ stemmten sich vehement dagegen. Ein Resultat zugunsten der Therapie mit Ginkgo-biloba-Extrakt passte nicht zu deren Generallinie. Die Arbeit wurde zurückgewiesen unter dem Vorwand, dass die Problematik den viel beschäftigten Vertragsärzten zu fern läge. Für eben diesen Leserkreis erscheint die Publikation in diesen Wochen in einer unabhängigen, anzeigenfreien Ärzte-Fachzeitschrift.
Solche Erfahrungen erinnern daran, dass nicht alle, die im Umfeld der Phytotherapie nach Behandlungsrisiken fragen, nur deren Vorsorge im Sinn haben. Bei Anwendungsgebieten, wie sie für die Phytotherapie typisch sind, werden Sicherheit und Akzeptanz der Präparate von Fachkreisen und Patienten zunehmend höher bewertet als die klinisch messbaren Effektstärken der Wirkstoffe. Verbunden damit verlagert sich derzeit zum Beispiel bei Erkrankungen wie Demenz oder Depression der Wettbewerb zwischen den Wirkstoffen eher weg von der Wirksamkeit und hin zur Verträglichkeit. Studien und Stellungnahmen zur Pharmakovigilanz werden deshalb in besonderem Maße für die Phytotherapie zu einem zentralen und in manchen Fällen sogar existenziellen Anliegen. Der gute Ruf im Sinne der besonderen Verträglichkeit pflanzlicher Arzneimitteln ist energisch und mit neuen Ideen zu verteidigen. Der bevorstehende 5. gemeinsame Berliner Phytotherapie-Kongress möchte auch dafür ein effizientes Forum bieten.

Volker Schulz

\section{LITERATUR}

1 Arzneimittelkommission der deutschen Ärzteschaft (AKdÄ): Pharmakovigilanz. AVP-Sonderheft 2005: 1-32. Eigenverlag der AKdÄ.

2 Schwabe U, Paffrath D: Arzneiverordnungs-Report 2004. Berlin, Heidelberg, New York: Springer; 2005. 